\title{
Videojuegos para el desarrollo del pensamiento crítico y el diálogo
}

\author{
Alexandra Ranzolin
}

\author{
Recibido: 10.05.2020 — Aceptado: 10.06.2020
}

\section{Title / Titre / Titolo}

Video games for the development of critical thinking and dialogue Les jeux vidéo pour le développement de la pensée critique et du dialogue Videogiochi per lo sviluppo del pensiero critico e del dialogo

\section{Resumen / Abstract / Résumé / Riassunto}

El presente artículo retoma los resultados de la investigación realizada por la autora, cuyo título es Videojuegos en el aula: una propuesta asociada al desarrollo del pensamiento crítico. Las inquietudes generadas en el estudio guardan relación con los lineamientos para desarrollar una propuesta de uso de los videojuegos en el aula, asociada al desarrollo del pensamiento crítico, en un grupo de jóvenes venezolanos escolarizados con edades comprendidas entre 13 y 17 años. Se procuró entender el videojuego como producto cultural en el contexto venezolano, los hábitos de consumo por parte de los sujetos estudiados y su relación con el pensamiento crítico y el desarrollo de procesos de diálogo. Se parte del análisis de las cuatro dimensiones propuestas por Lipman para el estudio del pensamiento crítico y se tomó en cuenta la concepción sociocultural del aprendizaje y la teoría de la Zona de Desarrollo Próximo de Vigotsky. Desde el punto de vista metodológico, se empleó la entrevista como técnica para la recolección de datos y se trabajó con una muestra intencional, conformada por dos adolescentes de 14 años, quienes interactuaron con videojuegos de distintos géneros. Se observó que el diálogo y la colaboración ante estas tecnologías son conductas espontáneas y necesarias para favorecer la resolución de problemas y la toma de decisiones. También los lineamientos de Lipman y Vigotsky son formulaciones que orientan las reflexiones en torno a las posibilidades que la alfabetización en los medios pueden ofrecer al desarrollo de miradas críticas sobre éstos.

This article takes up the results of the research carried out by the author in her study Video games in the classroom: a proposal associated with the development of critical thinking. Some of the concerns generated in the study are related to the guidelines for developing a proposal for the use of video games in the classroom, associated with the development of critical thinking, in a group of young Venezuelans in school between the ages of 13 and 17. We tried to understand video games as a cultural product in the Venezuelan context, the habits of video game consumption by the subjects studied and their relationship with critical thinking, and the development of dialogue processes. The starting point is the analysis of the four dimensions proposed by Lipman for the study of critical thinking and the sociocultural conception of learning together with Vigotsky's theory of Zone of Proximal Development. From the methodological point of view, the interview was used as a technique for data collection and work was done with an intentional sample, made up of two 14-year-old adolescents, who interacted with video games of different genres. It was observed that dialogue and collaboration in the face of these technologies are spontaneous and necessary behaviors to favor problem-solving and decision-making; thus, the guidelines proposed by Lipman and Vigotsky are formulations that allow to orient reflections on the possibilities that media literacy can offer for the development of critical views of the media.

Cet article reprend les résultats des recherches menées par l'auteur de cet article intitulé Les jeux vidéo en classe : une proposition associée au développement de la pensée critique. Certaines des préoccupations soulevées dans l'étude sont liées aux lignes directrices pour l'élaboration d'une proposition d'utilisation des jeux vidéo en classe, associée au développement de la pensée critique, dans un groupe de jeunes vénézuéliens scolarisés entre 13 et 17 ans. Nous avons essayé de comprendre les jeux vidéo en tant que produit culturel dans le contexte vénézuélien, les habitudes de consommation de jeux vidéo des sujets étudiés et leur relation avec la pensée critique et le développement de processus de dialogue. Le point de départ est l'analyse des quatre dimensions proposées par Lipman pour l'étude de la pensée critique et la conception socioculturelle de l'apprentissage et la théorie de la zone de développement proximal de Vigotsky. Du point de vue méthodologique, l'interview a été utilisée comme une technique de collecte de données et le travail a été effectué avec un échantillon intentionnel, composé de deux adolescents de 14 ans, qui ont interagi avec des jeux vidéo de différents genres. Il a été observé que le dialogue et la collaboration face à ces technologies sont des comportements spontanés et nécessaires pour favoriser la résolution des problèmes et la prise de décision ; cependant, l'importance de la sensibilisation à ces processus est prise en compte ; ainsi, les lignes directrices proposées par Lipman et Vigotsky sont des formulations qui permettent d'orienter les réflexions sur les possibilités que l'éducation aux médias peut offrir pour le développement d'un regard critique sur les médias.

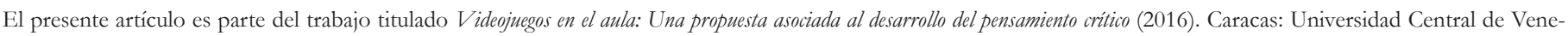

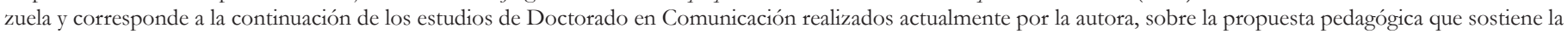
educación en medios asociada al constructivismo. 
Questo articolo riprende i risultati della ricerca condotta dall'autrice dal titolo Videogiochi in aula: una proposta associata allo sviluppo del pensiero critico. Alcune delle preoccupazioni generate nello studio sono legate alle linee guida per lo sviluppo di una proposta per l'uso dei videogiochi in classe, associata allo sviluppo del pensiero critico, in un gruppo di scolari venezuelani tra i 13 e i 17 anni. D'altra parte, abbiamo cercato di capire i videogiochi come un prodotto culturale nel contesto venezuelano, così come le abitudini di consumo di videogiochi da parte dei soggetti studiati e il loro rapporto con il pensiero critico e lo sviluppo dei processi di dialogo. Il punto di partenza è l'analisi delle quattro dimensioni proposte da Lipman per lo studio del pensiero critico e si è tenuto conto della concezione socio-culturale dell'apprendimento e della teoria della Zona di Sviluppo Prossimale di Vigotsky. Dal punto di vista metodologico, l'intervista è stata utilizzata come tecnica di raccolta dati e il lavoro è stato svolto con un campione intenzionale, composto da due adolescenti di 14 anni, che hanno interagito con videogiochi di diversi generi. Si è osservato che il dialogo e la collaborazione di fronte a queste tecnologie sono comportamenti spontanei e necessari per favorire la risoluzione dei problemi e il processo decisionale. Si osserva quindi che le linee guida proposte da Lipman e Vigotsky sono formulazioni che permettono di orientare la riflessione sulle possibilità che l'alfabetizzazione mediatica può offrire per lo sviluppo di punti di vista critici sui media.

\section{Palabras clave / Keywords I Mots-clé / Parole chiave}

Constructivismo, educación en medios, pensamiento crítico, videojuegos, generación $\mathrm{Z}$.

Constructivism, media education, critical thinking, video games, generation $Z$.

Constructivisme, éducation aux médias, esprit critique, jeux video, génération $\mathrm{Z}$.

Costruttivismo, educazione ai media, pensiero critico, videogiochi, generazione $Z$.

\section{Introducción}

\section{Una generación entre generaciones}

Una generación se está imponiendo, son los nacidos aproximadamente en el año 1995, quienes para el momento de la redacción del presente artículo cuentan con alrededor de 24 años. A estos sujetos se les denomina la Generación Z y, a diferencia de la Generación Y o Millennial, gran parte de su vida gira en torno a Internet y a las redes sociales. Aún con las diferencias que imponen los factores socioculturales, es común afirmar que para los $Z$ el mercado laboral es más desafiante, por lo que se han convertido en jóvenes emprendedores y orientados a abrir negocios propios. Prefieren, según Ortega, tecnología que facilite la comunicación a través de las redes, pues parten de la idea de que la interactividad y la comunicación interpersonal a través de las TIC — tecnología de la información y la comunicación- son un factor esencial para mantenerse informados, dependiendo aún más de estas tecnologías y de la conectividad a Internet. Sin embargo, según Ortega (11), «estamos ante la generación con más acceso a la información de la historia, pero no necesariamente la mejor informada». Por otra parte, los Jóvenes $Z$ no se limitan a lo local, sus equipos de trabajo y sus áreas de interrelación tienen como frontera el espacio global. Así también, esta generación está acostumbrada al cambio y la velocidad, por lo que no necesariamente hay tiempo para profundizar en temas o realidades, aunque podrían desarrollarse nuevas habilidades cognitivas para el procesamiento de información (Ortega). El presente artículo pone su foco en esta generación y cómo las TIC, y específicamente los videojuegos, se han convertido en un importante factor de desarrollo en su vida $y$, en particular, de habilidades de pensamiento crítico y diálogo.

Para la Generación Z los videojuegos constituyen el décimo arte, tecnología, medios de comunicación, espacios de entretenimiento y desarrollo de habilidades motoras y cognitivas. Los nacidos a partir de 1995 definitivamente se expresan bajo nuevos sistemas de signos y símbolos, así como también observan el mundo e interpretan la realidad de un modo distinto, disfrutan de la interactividad y de la jugabilidad, mecánicas que otras generaciones realizaban naturalmente de forma presencial en sus actividades lúdicas.

Uno de los aspectos que conviene tomar en cuenta de los videojuegos es su función original, a saber, su posibilidad esencial de entretener y luego de educar e informar. Son las consecuencias de lo que Huizinga (12) señala: «el ser vivo obedece cuando juega o (...) se ejercita para actividades serias que la vida le pedirá más adelante». Queda en evidencia, según el autor, que a tra- 
vés de lo lúdico se aprende y se desarrollan habilidades de diferente naturaleza.

El videojuego como entretenimiento es un camino para encontrar nuevos mundos, plenos de retos, los mismos desafíos que encontró Mario al intentar rescatar a la Princesa Peach ${ }^{1}$. Frasca, desarrollador de videojuegos, en el portal Elige Educar, afirma «los niños pueden aprender sin sufrir. Ellos saben de forma innata que la mejor manera de aprender es jugando».

Así, paralelo al entretenimiento, una vez más se reafirma la consistencia positiva de los videojuegos en el hecho educativo:

Los jóvenes consumidores de videojuegos perciben que su práctica lúdica tiene además componentes pedagógicos no esperados, que sí son capaces de identificar y explicitar. Entendemos, por lo tanto, que son conscientes del potencial educativo de los juegos que consumen y que utilizan, sin saberlo, como herramientas de educación informal (Gómez-García, Planells de la Maza, Chicharro-Merayo, 2017, p. 52).

Por otra parte, los videojuegos pueden considerarse importantes medios de comunicación, porque son fuente de transferencia, análisis, lectura cultural y de la realidad, además de refuerzo de las actitudes, gracias, en gran parte, al rasgo interactivo de los mismos que se traduce en intercambio de significados de forma presencial o en red. Todos estos procesos se desarrollan de manera inconsciente, a menos que intervengan instituciones como la familia o la escuela, entre otros, mediadores en los procesos de interacción con estas tecnologías.

Pasquali menciona la importancia de entender la comunicación como el espacio para hacer comunidad, palabras que exponen, etimológicamente, el vocablo común. El hacer comunidad es elemento clave en la construcción de realidades sostenibles que apunten a objetivos orientados al bien común a través del diálogo. La generación $Z$ se mueve en el plano tecnológico para descubrir significaciones y comprender la realidad circundan-

En el videojuego que lleva su nombre, Mario Bros debe superar siete mundos para encontrar a la princesa. Cuando llega al castillo del octavo mundo y Bowser es derrotado, Mario o Luigi liberan a la Princesa Peach. te, parece necesario entender estas nuevas formas de interrelación y de percepción del mundo.

Vale la pena destacar que la educación en medios como interdisciplina que reúne los conocimientos que provienen de la educación y la comunicación, fortalece la formación de miradas críticas sobre los contenidos que presentan los medios de comunicación. Según Hernández la educación en medios tiene como propósito «diseñar y aplicar estrategias pedagógicas sobre los medios —educación mediática- y mediaciones sociales en el sistema escolar formal, universidades y ambientes educativos no formales». De acuerdo con lo anterior, se hace presente un espacio formativo para la mayor comprensión y disfrute de las tecnologías y contenidos comunicacionales por parte de generaciones como la $Z$.

Los objetivos de este trabajo son establecer relaciones entre algunos lineamientos del pensamiento constructivista de Vigotsky y la educación en medios y analizar las prácticas de consumo de los videojuegos por parte de los jóvenes y su relación con el pensamiento crítico y el desarrollo de procesos de diálogo.

\section{Para el desarrollo de la inteligencia, el desarrollo del pensamiento crítico}

Señala Boisvert que una de las principales preocupaciones relacionadas con la educación según Mattew Lipman, Robert Ennis, John E. McPeck, Richard W. Paul y Harvey Siegel, guarda relación con la poca disciplina en el uso del pensamiento y la razón al elaborar conclusiones o reflexionar sobre hechos provenientes de contextos particulares. En este sentido, resalta Boisvert, la incapacidad de evaluar sobre la base de criterios y mostrar disposición para ejecutar acciones en función de dichas valoraciones como parte del proceso de desarrollo del pensamiento crítico. Así mismo, Gee señala en su obra que uno de los 36 principios a tomar en cuenta en relación con lo que se puede desarrollar a través de los videojuegos es, justamente, el pensamiento activo y crítico. Afirma Gee (49): 
«All aspects of the learning environment (including ways in which the semiotic domain is designed and presented) are setup to encourage active and critical, not passive, learning» ${ }^{2}$.

De acuerdo con lo anterior, la principal preocupación de Lipman (1998) se tradujo en sus observaciones en el superficial aprovechamiento de las oportunidades de estudio por parte de los alumnos norteamericanos. Estos se preparaban para las materias en secundaria únicamente para aprobar los exámenes, luego de los cuales olvidaban los contenidos aprendidos. De allí la necesidad de iniciar investigaciones que favorecieran los procesos de aprendizaje entre adolescentes y jóvenes. En tal sentido, el autor creó un método conocido como Filosofía para niños, orientado a favorecer la formación de una estructura crítica de pensamiento en niños, convencido de la necesidad de formar sociedades libres y solidarias. De esta forma, entiende también el diálogo y el acuerdo como pilares del proceso de pensar por sí mismos.

Por otra parte, uno de los propósitos de Lipman es construir sociedades maduras, capaces de asumir responsabilidades sobre sus decisiones, aspectos que se aprenden en ámbitos educativos formales y no formales según indica la tradición pedagógica. La concepción de ciertos términos y realidades sociales ponen en evidencia la infantilización de la sociedad ¿Puede aplicarse esto al acercamiento a la tecnología por parte de niños y adolescentes? Ya han señalado autores como Ortega (2016) el poco criterio de selección y procesamiento de información que se ha observado por parte de los jóvenes $Z$ al estar en contacto con las TIC, poniendo de manifiesto la necesidad de una educación en medios facilitada por los miembros de instituciones formadas en la materia, como la escuela y la familia.

Al igual que Vigotsky, aportará un elemento central en su discusión sobre el pensamiento crítico y es la relación con el contexto en el que se realizan las reflexiones y diálogos que permiten alcanzar los juicios sobre las diversas circunstancias y realidades. Para el autor el

2 Todos los aspectos del entorno de aprendizaje (incluidas las formas en que se diseña y presenta el dominio semiótico) se configuran para fomentar el aprendizaje activo y crítico, no pasivo (Gee, 2014, p. 49). Traducción realizada por la autora. pensamiento crítico se mueve en cuatro dimensiones ya que «es un pensamiento que 1) facilita el juicio porque 2) se basa en criterios 3) es autocorrectivo y 4) sensible al contexto» (Lipman, 1998: 174).

Conviene retomar en este apartado una afirmación de Masterman (1993: 90):

La labor realmente importante y difícil, del profesor de medios consiste en desarrollar suficientemente en los alumnos la confianza en sí mismos y la madurez crítica para que sean capaces de aplicar juicios críticos a los documentos de los textos que encuentren en el futuro. La dura prueba de cualquier programa de educación audiovisual es comprobar la medida en que los alumnos son críticos en la utilización y comprensión de los medios cuando el profesor no está delante. El objetivo primordial no es simplemente conocimiento y comprensión críticos, es la autonomia crítica.

La autonomía crítica de Masterman puede ser concebida como el resultado de un método que utiliza los medios audiovisuales para el desarrollo de una mirada sobre la realidad, compleja, en la que se desenvuelve el estudiante. En este sentido, lo que se aprende desde la educación para medios - pensamiento crítico, capacidad de diálogo, etc. - se intuye como posibilidad para la vida en una suerte de transferencia sistemática e intencional.

\section{Vigotsky visto desde la Educación para Medios: el contexto y el diálogo}

Uno de los autores que orienta sus reflexiones pedagógicas haciendo hincapié en las teorías sociales es Lev Vigotsky. Es de su autoría la concepción de la Zona de Desarrollo Próximo - ZDP_, que involucra la interacción como elemento fundamental para la resolución de problemas. Expone Pastor (2013) que si bien en la época de Vigotsky la interacción era cara a cara, las exigencias actuales requieren pensar en posibilidades de desarrollo de pensamiento crítico a través de tecnologías digitales tales como los videojuegos. 
Entre las aportaciones más significativas de Vigotsky se encuentran sus reflexiones en torno al pensamiento y el lenguaje. Él observó que el niño era capaz de desarrollar funciones mentales por sus propios medios e iniciativas, designando a esto la definición de nivel evolutivo real. Entre los hallazgos de Vigotsky se encuentra el hecho de que el niño era capaz de resolver situaciones o problemas con la ayuda de otros, entendiendo que también coexiste un nivel de desarrollo potencial. A esta distancia entre la capacidad de resolver un problema de manera independiente y la resolución del mismo con la ayuda de un colaborador más capaz, se le denominó Zona de Desarrollo Próximo - ZDP_ (Carrera y Mazzarella, 2001). De acuerdo con esto, el aprendizaje estimula y garantiza que florezcan procesos mentales.

Para Vigotsky un pilar del desarrollo cognitivo e integral es el hecho de que el ser humano se comprenda en un contexto que medie en su relación con el mundo y su realidad cultural. Es por esto que Carrera y Mazzarella (2001) señalan la importancia de asumir la relación entre el aprendizaje y el desarrollo, pues el niño, al entrar al sistema escolar, lleva consigo historia y aprendizajes previos que deben ser tomados en cuenta para el análisis, la toma de decisiones, la resolución de problemas y su desarrollo en general.

En relación con lo anterior, la educación para medios apela constantemente a la necesidad de encontrar al sujeto con su espacio, su tiempo y su realidad. Es lo que Orozco (1997) ha denominado mediaciones ${ }^{3}$, y que no se limita solo a un contexto, sino a múltiples espacios que marcan las modalidades de comprensión y representación de la realidad que asume el individuo en sus distintas etapas de desarrollo. Estas mediaciones están conformadas por instituciones, relaciones familiares o sociales, las tecnologías con las que están familiarizados e interactúa normalmente, la cultura en la que están inmersos, entre otros. Son influencias que Lipman (1998) ya

3 Ya acuñado por Martín Barbero y que comprende el sentido de la comunicación como un espacio de interacción entre quien comunica y quien recibe el mensaje, «fue así como la comunicación se nos tornó cuestión de mediaciones más que de medios, cuestión de cultura y, por tanto, no sólo de conocimientos sino de reconocimiento» (Martín Barbero, 1987: 10). había denominado comunidades de investigación, y que se entienden como espacios confortables y libres en los que el niño y el joven dialogan y participan para descubrir criterios y soluciones verdaderas a problemas reales.

De acuerdo con lo que plantean autores como Vigotsky y Orozco, al hablar de mediaciones, lo que se propone es desmitificar aspectos que se han tratado como tabú dentro del propio contexto en el que se desenvuelve el sujeto, para convertirlos en objetos de aprendizaje. Así, por ejemplo, el generar diálogo y acompañamiento frente al uso de las tecnologías de la comunicación e información, permite el establecimiento de criterios para su máximo aprovechamiento en relación con el desarrollo de habilidades y comportamientos que favorezcan el desarrollo del pensamiento crítico, esto implica también un viraje en el modo de concebir la educación.

Desde esta perspectiva, las personas en permanente diálogo y conscientes del contexto en el que se encuentran, según Orozco (1997: 27), son «sujetos capaces de tomar distancia de los medios y sus mensajes, pero también sujetos ansiosos de encontrar en ellos lo espectacular, lo novedoso, lo insólito, todo eso que nos emocione, nos estremezca, nos divierta y nos haga salir, aunque sea por momentos, de nuestra rutina y existencia cotidiana».

Hasta este momento, los aportes de Vigotsky apuntan a comprender la interacción social como base en el proceso de aprendizaje. De hecho, en el estudio realizado por Ranzolin (2016), se observó que de la muestra de 232 adolescentes que participaron respondiendo el cuestionario diseñado para la investigación, el 78\% expresó su preferencia por jugar videojuegos en compañía. Más adelante, en este artículo, se podrá observar esta relación observada durante la fase cualitativa del estudio.

\section{Método}

La metodología aplicada en la etapa del estudio que se presenta en este artículo fue de tipo cualitativa, descriptiva y de campo. A partir de esta definición, se reali- 
zaron seis (6) entrevistas semiestructuradas, de $30 \mathrm{mi}-$ nutos cada una, a una muestra intencional conformada por dos (2) adolescentes, hermanas gemelas, de 14 años, estudiantes del tercer año de Educación Media General, en una escuela privada de la ciudad de Caracas, luego de jugar videojuegos, compartiendo el mismo espacio físico — su hogar - y durante igual cantidad de tiempo -20 minutos-. Adicionalmente, las gemelas pertenecían a una familia conformada por su madre, padre y dos hermanas, quienes también se involucraron como espectadoras de las participantes en el estudio - 12 y 8 años- Las entrevistas se realizaron a lo largo de seis sesiones -una por semana-, en su hogar.

Los géneros de los videojuegos empleados fueron estrategia - Fire Emblem —, mundo abierto y construcción -Minecraft_, deportes - Wii Sport Resort-, terror — Silent Hill_ , disparo en primera persona —Deer Hunter - y rompecabezas —94 segundos_- A través de la realización de las entrevistas se obtuvieron datos que contribuyeron a interpretar la información recogida por medio de los cuestionarios y que, aunque no puede generalizarse, favorecieron el cruce de datos y la detección de coincidencias entre los elementos obtenidos a partir de ambas metodologías - cuantitativa y cualitativa, esta última el centro del presente artículo-.

Entre los juegos señalados como 'más divertidos' por las adolescentes participantes en el estudio, se observó preferencia por Minecraft, Wii Suport Resort y Silent Hill, especialmente por su jugabilidad y posibilidad de interacción. Por otra parte, de acuerdo con lo señalado en las entrevistas correspondientes, Deer Hunter fue considerado como un videojuego 'poco atractivo' por ser predecible y no presentar suficientes retos, así como Fire Emblem por no haberse podido instalar en el equipo. Los dispositivos utilizados para la realización de la experiencia fueron celulares inteligentes, laptops y una consola de Wii. Para el momento, las adolescentes participantes en el estudio habían jugado videojuegos frecuentemente con estos equipos, siendo la primera interacción de los sujetos con los videojuegos a través de la consola de Wii. Las adolescentes, pertenecientes por edad a la generación Z, presentaron rasgos distintivos de su época, entre ellos, la facilidad para la localización de información en línea como forma de conocer la realidad a la que se aproximaban y gusto por las aplicaciones interactivas como los videojuegos. Sin embargo, vale la pena destacar que el acceso a esta tecnología no siempre es estable y accesible en el contexto venezolano. ${ }^{4}$

Como categorías de análisis frente a la experiencia señalada, se retomaron las dimensiones de Lipman reflejadas en los apartados anteriores. Se procuró detectar habilidades para: 1) La resolución de problemas —orientadas a la localización de información para la solución de situaciones-; 2) El análisis — habilidad a través de la cual se pueden reconocen los componentes de un todo, hasta observar sus principios sustanciales y reagruparlos para establecer relaciones-; 3) La toma de decisiones — destinada a la elección entre dos o más opciones-. Se destaca que la finalidad última de este procedimiento fue detectar la emisión de juicios a partir de criterios, es decir, el resultado del ejercicio del pensamiento crítico según Lipman partir del diálogo y la conformación de comunidades de investigación.

\section{Análisis y resultados}

La investigación se enfocó en las cuatro dimensiones propuestas por Lipman: autocorrección, sensibilidad al contexto, orientados por criterios y juicios razonables, con la finalidad de valorar la presencia de componentes del pensamiento crítico en la utilización de videojuegos por parte de las adolescentes participantes del estudio. Al elaborar el presente artículo, los resultados obtenidos en el estudio inicial fueron leídos a la luz de las reflexiones realizadas por Vigotsky. El foco se ubicó en la mirada socio cultural para el aprendizaje y la impor-

\footnotetext{
4 Por ejemplo, la CEPAL mostró que Venezuela presentaba «la velocidad más lenta en el acceso a través de la banda ancha fija (1,9 Mbps). En cuanto al rendimiento, el país es uno de los más rezagados con solo $0,5 \%$ de conexiones de más de $10 \mathrm{Mbps}$ y $0,2 \%$ de conexiones por encima de $15 \mathrm{Mbps} »$. Señala el estudio que para 2015 todavía más de la mitad de la población no contaba con el servicio de conexión a Internet, había una importante participación en redes sociales y se marcaban diferencias de acceso entre las zonas rurales y las urbanas.
} 
tancia de la Zona de Desarrollo Próximo —ZDP_, como componentes de las propuestas educomunicativas vinculadas al uso de los videojuegos.

Para Lipman las distintas dimensiones de análisis presentadas se definen de la siguiente manera:

Tabla 1: Dimensiones de pensamiento crítico (Lipman, 1998).

\begin{tabular}{|c|}
\hline Autocorrección \\
\hline $\begin{array}{l}\text { Capacidad de encontrar errores en la propia forma de pensar o } \\
\text { en la de otras personas, de clarificar ambigüedades o de detectar } \\
\text { inconsistencias. }\end{array}$ \\
\hline Sensibilidad al contexto \\
\hline $\begin{array}{l}\text { Observación de significado a partir de matices culturales o pun- } \\
\text { tos de vista, el reconocimiento de diferencias por áreas de co- } \\
\text { nocimiento o interpretaciones, la precisión en las traducciones, } \\
\text { el énfasis o variaciones en las intenciones de los hablantes, entre } \\
\text { otros. }\end{array}$ \\
\hline Orientación por criterios \\
\hline $\begin{array}{l}\text { Identificación de las conductas que guardan relación con la pre- } \\
\text { sencia de valores compartidos, convenciones, esquemas com- } \\
\text { parativos comunes, requisitos, perspectivas, principios, reglas, } \\
\text { estándares, definiciones, hechos, pruebas y propósitos. }\end{array}$ \\
\hline Juicios Razonables \\
\hline $\begin{array}{l}\text { Observación de acuerdos tras deliberaciones, veredictos tras } \\
\text { ensayos o encuestas, decisiones, determinaciones, soluciones a } \\
\text { problemas reales o teóricos, clasificaciones o categorizaciones, } \\
\text { evaluaciones, distinciones, conexiones y deliberaciones. }\end{array}$ \\
\hline
\end{tabular}

Fuente: Elaboración propia.

En relación con las cuatro dimensiones antes descritas, en los resultados del estudio destacan algunos indicadores que manifiestan la presencia de conductas sobre cada una. En primer lugar, la dimensión autocorrección apela a indicadores como «situar errores en el pensamiento de los otros», «son conscientes de los errores de su propio pensamiento»y «piden razones y criterios cuando no se han dado». Por otra parte, la segunda dimensión, entendida como sensibilidad al contexto se tradujo en indicadores conocidos como «observan que los significados de las definiciones se modifican en función al contexto» y «notan diferencias entre las situaciones actuales y situaciones pasadas aparentemente semejantes». Así mismo, de la dimensión orientado por criterios se observaron los indicadores valores compartidos, convenciones, esquemas comparativos comunes, requisitos, principios y hechos; $y$, finalmente, con respecto a la dimensión juicios razonables se evidenció la presencia de valores y la valoración de acciones de los personajes.

En los indicadores correspondientes a la dimensión autocorrección entendido como «situar errores en el pensamiento de los otros»y «son conscientes de los errores de su propio pensamiento» (Lipman), se ofrecieron como evidencias que las adolescentes observaron entre sí la no comprensión o la realización errada de algunas de las acciones y procedimientos que se presentaban en los videojuegos ejecutados. Se manifestó, en este caso, la invitación a resolver las dificultades presentadas para las adolescentes y para los observadores externos - hermanas-, los cuales intervinieron libre y activamente para intentar solucionar y aclarar dudas a través de conversaciones y no por la intervención física de los dispositivos utilizados ante estos procedimientos.

Así mismo, se destacó la toma de conciencia de los errores de su propio pensamiento al realizarse la instalación del software de cada videojuego utilizado - una de los primeros pasos para alcanzar el disfrute del mismo-, así como la facilidad de ejecutar operaciones y probar varias opciones para canalizar la detección de fallas de pensamiento. Por otra parte, las adolescentes pudieron realizar diversas acciones de forma independiente, comprobando que éstas dominan diversos procesos y son capaces de corregirse a su debido momento.

Lo anterior fue evidente al momento de intentar instalar el videojuego Fire Emblem, en la que se observó la siguiente conversación obtenida durante el proceso y que muestra la detección del error y la autocorrección, así como la indicación para que se busque la solución al problema:

Y pide ayuda, X la niega y comenta: «yo tuve que averiguarlo, averígualo tú también» (Ranzolin, 2016).

Así mismo, las adolescentes mostraron capacidad para alternar, frente a las situaciones que se presenta- 
ron, el desarrollo de operaciones, apreciándose la selección de alternativas, manipulación de equipos, redes y contenidos en línea; del mismo modo, y de cara al diálogo expuesto anteriormente, es posible ver esta alternancia en las operaciones una vez que no se mostraron errores o dificultadas en las acciones ejecutadas con anterioridad. Lo expuesto se evidencia a través del siguiente comentario:

Y: Yo me metí en una red social para ver fotos mientras se instalaba (Ranzolin, 2016).

En relación con si piden razones o criterios cuando no se han dado, las adolescentes solicitaron explicaciones al director de la actividad al desconocer alguna respuesta, o reconocían las intenciones del fabricante del software. Fue evidente la necesidad del ejercicio de diálogo, la presencia de espectadores o adultos significativos, que con sus opiniones favorecían o no la obtención de lineamientos. Este diálogo permitió la deducción de criterios, la práctica a través de la intuición y la utilización de la razón frente a la emoción - explicitado por los sujetos - para la resolución de acertijos y suposiciones. Se señala que el medio audiovisual domina la respuesta emocional porque engancha a partir del poder de la imagen, sin embargo, el videojuego parece exigir algo más que respuestas basadas en impulsos según se testimonia en esta experiencia.

Fueron de interés diálogos, casi poéticos, como el siguiente:

\section{Y: Miren hay estrellas}

¿Y hay una luna? Porque está todo oscuro y si no hay una antorcha...

X: Puedes salir trepándote a un árbol (Ranzolin, 2016).

En relación con la dimensión «sensibilidad al contexto», las adolescentes fueron capaces de detectar significados de definiciones que se ven modificados en función al contexto, se observó que no conocían el origen del videojuego, uno de los aspectos centrales para conocer el sentido del mismo. Quedó también en evidencia el desinterés por uno de los videojuegos, mas no por su origen y que, ante la falta de informa- ción sobre el mismo, se inició el proceso de inferencia a partir del diálogo. Por otra parte, ante el indicador sobre la identificación de diferencias en situaciones presentes y pasadas supuestamente semejantes, entendido como patrones y modelos repetidos a lo largo del juego que pueden cambiar bajo diversas condiciones, las adolescentes verbalizaron haber encontrado comandos del teclado que se repiten en varios videojuegos para realizar las mismas acciones. Finalmente, los sujetos reconocieron diversos sistemas de valores presentes en los videojuegos encontrando oportunidades en función de lo que podían otorgar los distintos elementos detectados.

\section{$\mathrm{V}:$ ¿Ustedes logran valorar a uno de los personajes? $\mathrm{X}$ : sí}

$\mathrm{V}:$ ¿De acuerdo a qué los valoran?

$\mathrm{X}$ : Yo los valoro. Por ejemplo, los clíper te pueden destruir las cosas pero cuando tú logras cazar uno y matarlo, ellos te dan pólvora y eso te ayuda (Ranzolin, 2016).

La evidencia de convenciones o acuerdos se alcanzó luego de la evaluación de conversaciones entre los sujetos de la investigación, sobre distintos elementos como personajes, situaciones, entre otros, estimando la relación entre estos, nuevamente gracias a procesos de diálogo.

Uno de los aspectos más interesantes surgidos de esta experiencia es el reafirmar el hecho de la importancia del intercambio de ideas casi natural entre quienes juegan videojuegos. Lo anterior rompe el mito del aislamiento entre quienes disfrutan de esta tecnología. Resulta de mayor interés, cómo esta realidad se presenta con mayor intensidad cuando interviene un adulto significativo - es decir, con influencia o relevancia para la vida del joven - lo que refuerza la teoría de las mediaciones expresada por Orozco ${ }^{5}$ y los supuestos de

\footnotetext{
5 Señala Orozco que «el «juego de la mediación múltiple», tanto en los medios, como en las audiencias, como en sus procesos de recepción, es lo que finalmente define lo que los medios logran, y lo que las audiencias se apropian, negocian o rechazan de los medios, así como el uso que hacen de ellos. Más que en la emisión, es justamente en el «largo y complejo» proceso de la recepción, donde se produce la comunicación, donde se le da su sentido. Un sentido que no es autónomo completamente de lo propuesto por los medios, pero que tampoco está restringido a eso» (Orozco, 1997, p. 28).
} 
Vigotsky sobre la Zona de Desarrollo Próximo, en el caso de la compañía de pares al realizar ciertas operaciones.

Es característica de este indicador la siguiente comunicación:

V: ¿Había condiciones para avanzar en el juego?

$\mathrm{Y}: \mathrm{No}$

$\mathrm{X}$ : Ganar

Y: Es decir, el juego te dice cómo vas avanzando y si pierdes pierdes puntos de avance (Ranzolin, 2016).

En cuanto al tercer indicador, entendido como «esquemas comparativos comunes» de Lipman (1998), fue clara la presencia de comparaciones de un videojuego con otro utilizado previamente, se observaron relaciones sobre las posibilidades de diversión o entretenimiento, exigencia y jugabilidad.

A continuación, una conversación que ejemplifica el indicador anterior:

$\mathrm{V}$ : ¿Con qué juego pueden comparar esta experiencia?

$\mathrm{X}$ : Con $A$ Pensar solo que con tiempo.

$\mathrm{V}$ : ¿Cuál es más divertido?

Y: Este, aunque es aburrido si lo juegas sola (Ranzolin, 2016).

El tema de comprender los requisitos para jugar videojuegos por parte de las adolescentes se presentó a partir de la solicitud de instrucciones o reglas de juego por parte de las videojugadoras, así como de las condiciones para avanzar en el juego. Sin embargo, luego fue notoria la disminución del interés por videojugar al tener que consultar las instrucciones, la ansiedad por jugar más que por entender las instrucciones, la necesidad de aclarar dudas para avanzar, la comparación entre distintos dispositivos de juegos y sus requisitos para avanzar, la importancia de actuar por instinto o impulsos cuando no hay reglas claras y el diálogo para comprender las reglas del juego, condición necesaria para progresar en el videojuego.

Una comunicación que ejemplifica este indicador es la siguiente:

\section{$\mathrm{V}:$ ¿Ya habían jugado este juego?}

$X$ y Y: Sí, pero nunca en computadora.

$\mathrm{V}$ : ¿Por qué les emociona tanto?

$\mathrm{X}$ : Porque en computadora es diferente y lo ves todo distinto.

Y: En la tabla no tienes todas estas funciones.

$\mathrm{X}$ : En la tabla hay reglas y en la computadora es todo sin control.

V: Entonces, ¿cómo sabes que tienes que actuar si no hay reglas?

Y: —silencio- por instinto.

$\mathrm{V}$ : ¿Qué es un instinto?

X y Y: Un impulso que te motiva a hacer algo, como matar gente — en el juego (Ranzolin, 2016).

La importancia de favorecer el uso de herramientas tecnológicas como los videojuegos con base en principios o fundamentos guarda relación con el hecho de resguardar el objetivo primero de la educación para los medios, el cual consiste, como ya se ha mencionado, en incentivar una actitud crítica ante los contenidos de los medios de comunicación.

Finalmente, el último indicador de la dimensión orientado por criterios corresponde a «hechos» (Lipman), el cual se tradujo en el uso de razones y no emociones para resolver problemas. Parece, en este apartado, que las adolescentes son capaces de diferenciar el pensamiento crítico de un pensamiento desordenado o desestructurado. Al igual que Lipman, Vigotsky aportará información de interés en relación con el hecho de la importancia de tomar conciencia y conocer el contexto para potenciar el aprendizaje y generar apoyos para alcanzar metas de conocimiento.

Este indicador puede ser ejemplificado por medio del siguiente diálogo:

V: Les preguntaba antes si ustedes estaban conscientes de que tomaban decisiones, porque parece que trabajaban más por impulsos e instintos, ¿'eso es así? ¿Qué era primero, el impulso y luego se daban cuenta de que estaban tomando una decisión o que estaban tomando decisiones y luego reaccionaban?

Y: no, no, no, primero tú, por ejemplo, ves una cabrita y dices «qué lindo el animalito» y luego «cónchale, esto me puede dar 
cuernos, me puede dar carne, vamos a matarlo, y vas y lo matas», pero hay momentos como cuando tienes un zombie atrás, que simplemente te vas a voltear y le vas a dar, no importa qué te dé lo vas a matar y listo, si no él te mata a ti (Ranzolin, 2016).

La última dimensión que ofrece Lipman para el estudio del pensamiento crítico, «juicios razonables», señala indicadores como «decisiones» $\mathrm{y}$ «distinciones». En tal sentido, se observó que las adolescentes eran capaces de realizar arreglos para jugar a través de procesos diálogo, y con ello demostrar que eran capaces de resolver problemas y tomar decisiones, realizar trabajo en equipo y como consecuencia, brindar apoyo mutuo. Avanzar en un juego parece mucho más difícil y menos gratificante si no se producen estos procesos comunicativos.

El diálogo que se presenta a continuación ilustra lo que se desea expresar:

Y: Me dijeron que se pueden construir montañas rusas.

$\mathrm{X}$ : Pero eso es en modo creativo.

Y: ¡Vamos a hacer Disney!

$\mathrm{X}$ : Vamos a hacer un estado en el que se encuentren Howards, Panen [sic] y la ciudad de Divergente que es Chicago, ¿qué más se puede encontrar allí?

Y: ¡Ah! La Tierra Media, el Callejón Diagonal... Falta poco para la instalación.

X: Mi casa va a tener flores (Ranzolin, 2016).

Un segundo indicador de la dimensión mencionada anteriormente, se refiere a la solución de problemas a través del cual se intentó evidenciar que las adolescentes reconocieran diferentes opciones para resolver una situación y tomaran decisiones con los participantes para enfrentarse a problemas a lo largo del juego. Fue de interés la búsqueda de alternativas mediante el diálogo y la verificación de que no siempre se contaba con alternativas frente a las situaciones problemáticas que presentaba el videojuego.

$\mathrm{V}:$ ¿Conversaron durante el juego para ponerse de acuerdo en algo?

$\mathrm{X}$ : En estos 20 min. no, pero con un poco más de tiempo yo le hubiese preguntado a Y cuando se hiciera de día en el juego, qué hizo para encontrar el pueblito.

\section{$\mathrm{V}$ : ¿Hubiesen hecho intercambios de información?}

Y: Hubiésemos hecho intercambios de información (Ranzolin, 2016).

El último indicador analizado para la dimensión juicios razonables, corresponde a «conexiones», a partir del cual se observó si las adolescentes expresaron que lo visto en el videojuego se expresaba o enlazaba con aspectos de la vida real. Las adolescentes expresaron vivencias como las siguientes:

\section{$\mathrm{V}$ : ¿El juego expresa cosas de la vida real?}

Y: Sí

$\mathrm{V}:$ ¿Por ejemplo?

Y: Por ejemplo, para mí Caracas es una selva y Minecraft es como una selva, tú quieres algo y lo consigues y haces lo que sea para conseguirlo.

$\mathrm{X}$ : yo creo que tiene factores de la vida real porque tienes que trabajar para conseguir lo que quieres, en este juego también, y siempre en la vida real van a haber obstáculos y siempre en la vida real hay obstáculos, no es que todo se hace fácil, y cuando juegas conectado tienes que negociar con la gente para que se hagan alianzas (Ranzolin, 2016).

Quedaron en evidencia procesos de comparación, así como valores y actividades compartidas entre el mundo real y el videojuego como posibilidad para la resolución de problemas y la toma de decisiones de situaciones reales.

\section{A modo de cierre}

La Generación $Z$ es la denominación que se le ha dado a un grupo de personas que han adoptado las TIC como herramienta de trabajo, entretenimiento, etc. No son cualquier conglomerado, conforman hoy, por ejemplo, adolescentes que viven de la interactividad y que, conscientes o no, están siendo formados en una manera de concebir la realidad y desarrollar sus propias habilidades cognitivas. Sin embargo, en países como Venezuela, en los que la brecha digital y el acceso limitado a las TIC es más evidente, puede entenderse también que la 
inclusión dentro de la categoría $Z$, en ocasiones depende más de la disponibilidad y aprovechamiento de la tecnología que de un rango de edad.

Una de las tecnologías con las que esta generación se entiende, disfruta y aprende, son los videojuegos, hoy señalados como nuevos medios de comunicación según Peláez $z^{6}$ y por la educación en medios como espacios en los que sus usuarios deben ser alfabetizados, ya que su aprovechamiento puede favorecer el aprendizaje y desarrollo integral del niño y el joven, así como la mirada crítica frente a los medios audiovisuales y digitales.

En el desarrollo del pensamiento crítico, uno de los objetivos esenciales de la educación en medios, requiere intencionalidad en su enseñanza y en su estudio. Los resultados de la presente investigación ponen en evidencia que las dos adolescentes participantes en la investigación que dio pie a este artículo hacen uso de las cuatro dimensiones propuestas por Lipman -autocorrección, sensibilidad al contexto, orientados por criterios y juicios razonables-, como expresiones del pensamiento crítico. Sin embargo, es importante tomar en cuenta el planteamiento de Orozco (2012) cuando señala que el problema de fondo con el uso de la tecnología — videojuegos - y el aprendizaje de estos procesos cognitivos, es la falta de toma de conciencia sobre su presencia y, por tanto, la incapacidad para apropiarse de estos y de transferirlos a otras esferas de la vida. Así, si se reconoce como un valor el diálogo y la cooperación para el cumplimiento de retos en los videojuegos, podría entenderse como hipótesis la posibilidad de que estas habilidades se transfieran, por ejemplo, a esferas sociales, escolares o familiares de la persona en contextos diversos.

Un aspecto que emerge en la investigación es la presencia de diálogo entre las adolescentes para la resolución de problemas, la toma de decisiones y el análisis de elementos del contexto, procesos que evidencian el pensamiento crítico. Como se ha señalado anterior-

«El videojuego, como medio masivo que es, también tiene la capacidad de llevar mensajes, expresar las ideas, críticas y consternaciones sociales/políticas de un autor o autores. A la vez que también es capaz de llevar toda una ideología como sucede en el cine o la televisión» (Peláez). mente, Vigotsky ofrece a la educación en medios una de las claves teóricas que influencian el desarrollo del pensamiento crítico y que también resalta Lipman al hablar de «sensibilidad al contexto». Esta clave guarda relación con la toma de conciencia por parte del ser humano de su incapacidad de permanecer aislado. Según lo que plantean Carrera y Mazzarella, para Vigotsky el entorno será siempre un factor determinante que permitirá alcanzar el aprendizaje y el esperado desarrollo de las máximas potencialidades de la persona.

La cooperación, gracias al diálogo y la sensibilidad hacia el otro — aunque no exista conciencia plena-, es garante de espacios de interacción para el desarrollo de prácticas que promuevan, por ejemplo, el desarrollo de pensamiento crítico. Este requiere sumirse en contextos y contenidos específicos para ser comprendido y estudiado. Vigotsky señalará que la Zona de Desarrollo Próximo - ZDP_ explica la posibilidad de resolución de un problema de manera independiente o con la ayuda de un colaborador más capaz. Los resultados del estudio desarrollado demuestran la interacción que entre los videojugadores favorece procesos de resolución de problemas y toma de decisiones, elementos a seguir profundizando en futuras investigaciones.

Finalmente, se entiende que investigaciones que asocian procesos complejos como el desarrollo del pensamiento crítico, requieren asumir variables múltiples y profundizar en contextos y contenidos concretos para su verificación. La educación en medios, a través de sus distintas propuestas, se ha adjudicado el reto de estudiar el ámbito comunicacional con la finalidad de formar «en los medios y con los medios de comunicación» (Ferrés), entendiendo que las bondades de esta educación, pedagogía y didáctica son transferibles en su esencia a otras esferas de la vida de la persona —entiéndase también la capacidad de diálogo—. Los medios facilitan el conocimiento y la interpretación de lo que acontece en el mundo. De allí la importancia de desmitificarlos y aprovechar sus bondades, así como de alfabetizar a las generaciones que son capaces de manipularlos de forma intuitiva. En este sentido ¿qué tienen que decir en concreto los que ya han reflexionado sobre estos te- 
mas, los que son parte de generaciones anteriores a, por ejemplo, los Jóvenes $Z$ ? Por último, la investigación que da pie a este artículo constituye parte de la profundización sobre el constructivismo como pilar pedagógico de la educación en medios. Así, también se ha iniciado la discusión con algunos lineamientos propuestos por Vigotsky, los cuales se asocian con la propuesta de Lipman sobre pensamiento crítico, especialmente en el área de sensibilidad al contexto y el aprendizaje. Conviene ir a fondo en estos temas y validar su pertinencia como criterio de análisis, así como actualizarlos como tradición pedagógica asociada a la educación en medios.

\section{Bibliografía}

BoIsvert, Jacques. La formación del pensamiento crítico: Teoría y práctica. México: Fondo de Cultura Económica, 2004.

Carrera, Beatriz y Clemen Mazzarella. «Vygotsky: enfoque sociocultural». Educere, 5(13), 2001, pp. 41-44. Recuperado de http:/ / www.redalyc.org/articulo.oa?id=35601309.

CEPAL. Estado de la banda ancha en América Latina y el Caribe 2016. Santiago: Naciones Unidas, 2016.

Frasca, Gonzalo. «Gonzalo Frasca, el uruguayo que diseña videojuegos con los que enseñan matemáticas en Finlandia». Elige educar, 2018. Recuperado el 25 de enero de 2018 de http://www.eligeeducar.cl/gonzalo-frasca-uruguayodisena-videojuegos-los-ensenan-matematicas-finlandia.

Ferrés, Joan. Educación y Televisión. Barcelona: Paidós, 1994.

GEe, James Paul. What Video Games Have to Teach Us About Learning and Literacy. EEUU: Macmillan, 2014.

Gómez-García, Salvador, Antonio José Planells de la Maza y Mar Chicharro-Merayo. «LLos alumnos quieren aprender con videojuegos? Lo que opinan sus usuarios del potencial educativo de este medio». Educar, 2017. Recuperado de
http://www.raco.cat/index.php/Educar/article/view/ 317270 .

HERnÁNDEZ, Gustavo. «Introducción a la teoría de la educación para los medios». Anuario ININCO. Investigaciones de la Comunicación, 13(1), 2001. Recuperado de http:/ /www.bantaba.ehu.es/formarse/ficheros/view/Introducci\%F3n_a_ la_Teoria_de_la_Educaci\%F3n_para_los_medios. pdf?revision_id=56379\&package_id $=56305$.

Huizinga, Johan. Homo Ludens. Madrid: Alianza, 2007.

Lipman, Matthew. Pensamiento complejo y educación. Madrid: Ediciones de la Torre, 1998.

Martín-Barbero, Jesús. De los medios a las mediaciones. Barcelona: Editorial Gustavo Gili, 1987.

Masterman, Len. La enseñanza de los medios de comunicación. Madrid: Ediciones de La Torre, 1993.

Orozco, Guillermo. «Medios, audiencias y mediaciones». Comunicar, 8, 1997. Recuperado de: http://www2.uned. es/ntedu/espanol/master/primero/modulos/teoria-dela-informacion-y-comunicacion-audiovisual/orozco.pdf.

—Educomunicación 2.0. UNED Entrevistas, 2012. Recuperado de: https://www.youtube.com/watch?v=JD8Di8qyv6g.

ORTEGA, Iñaki. Generación Z. El último salto generacional. Resumen ejecutivo. Bilbao: Universidad de Deusto, Atrevia, 2016.

Pasquali, Antonio. Comprender la comunicación. Caracas: Monte Ávila Latinoamericana C.A., 1990.

PAstor,JoséLuis.«Aprendizajesocialynuevas formasdeentender el proceso de enseñanza-aprendizaje». Padresy maestros, 351, 2013. Recuperado de http://revistas.upcomillas.es/ index.php/padresymaestros/article/view/1045/889.

PeláEz, Bruno. «Videojuegos: Una aproximación». Comunicología: Indicios y Conjeturas. México: Universidad Iberoamericana Ciudad de México, 2006.

RANZOLIN, Alexandra. Videojuegos en el aula: Una propuesta asociada al desarrollo del pensamiento crítico. Caracas: Universidad Central de Venezuela, 2016. 